\title{
Mental rotation of congenitally absent hands
}

\author{
MARION FUNK AND PETER BRUGGER \\ Department of Neurology, University Hospital Zurich, Zurich, Switzerland
}

(Received April 21, 2006; Final Revision June 15, 2007; AcCePted June 15, 2007)

\begin{abstract}
We compared motor imagery performance of normally limbed individuals with that of individuals with one or both hands missing since birth (i.e., hand amelia). To this aim, 14 unilaterally and 2 bilaterally amelic participants performed a task requiring the classification of hands depicted in different degrees of rotation as either a left or a right hand. On the same task, 24 normally limbed participants recapitulated previously reported effects; that is, that the hand motor dominance and, more generally, a lifelong use of hands are important determinants of left-right decisions. Unilaterally amelic participants responded slower to hands corresponding to their absent, compared with their existing, hand. Moreover, left and right hand amelic participants showed prolonged reaction times to hands (whether left or right) depicted in unnatural orientations compared with natural orientations. Among the bilateral amelics, the individual with phantom sensations, but not the one without, showed similar differentiation. These findings demonstrate that the visual recognition of a hand never physically developed is prolonged, but still modulated by different rotation angles. They are further compatible with the view that phantom limbs in hand amelia may constrain motor imagery as much as do amputation phantoms. (JINS, 2008, 14, 81-89.)
\end{abstract}

Keywords: Body scheme, Mental rotation, Hand laterality task, Phantom sensation, Amelia, Handedness

\section{INTRODUCTION}

Higher-order interactions between vision and bodily representations determine the mental rotation of body parts (Bonda et al., 1995; Kosslyn et al., 1998; Parsons, 1994; Shenton et al., 2004). That is, even the mere imagination of a limb in a specific posture or spatial orientation automatically invites a participation of the motor system. One of the most frequently used experimental paradigms in studies of the mental rotation of body parts is a task requiring laterality decisions to visually presented hands in different orientations. In this "hand laterality task," subjects have to decide if the presented hand is a left or a right limb (e.g., Parsons, 1987, 1994; Sekiyama, 1982). Reaction times (RTs) of correct decisions clearly reflect the involvement of the observers' motor system. First, right-handers recognize right hands faster than left hands, whereas left-handers do not show a comparably strong left-hand preference (Gentilucci et al., 1998). Second, backs of hands are faster classified than palms as long as the fingers are pointing up, medially, or laterally (Parsons, 1987). Third, right and left hands whose

Correspondence and reprint requests to: Marion Funk, Neuropsychology Unit, Department of Neurology, University Hospital Zurich, Frauenklinikstrasse 26, CH-8091 Zurich, Switzerland. E-mail: marion.funk@usz.ch fingers are pointing in a medial direction (i.e., toward the body's midsagittal plane) are faster responded to than those whose fingers are pointing laterally (i.e., away from the body's midsagittal plane; Parsons, 1994), a RT difference we henceforth designate the "medial-over-lateral advantage" (MOLA). All these observations suggest that, in recognizing static images of left and right hands in varying positions, subjects implicitly move their own hands into the respective position to reach a laterality decision. The "implicit reaching" (Parsons et al., 1995) toward the visual stimulus is not only reflected behaviorally (i.e., in prolonged RTs to awkward, unnatural display postures), but the involvement of the motor system has also been demonstrated in functional imaging studies (e.g., Bonda et al., 1995; Decety et al., 1994; Parsons et al., 1995).

Although specific impairments of hand laterality decisions were described after acquired hand motor dysfunction (e.g., Fiorio et al., 2006; Johnson et al., 2002), the impact of a complete absence of any hand motor activity since birth has received little attention. To date, only two studies set out to investigate hand laterality decision in amelic individuals, that is, individuals born without hands (bilateral hand amelia) or with only one hand (unilateral hand amelia). First, Brugger et al. (2000) assessed hand (and foot) laterality decisions of a woman (A.Z.) born without hands and 
feet, who nevertheless reported vivid phantom sensations of all missing limbs. From the fact that RTs were prolonged for body parts with fingers (toes) pointing down $\left(180^{\circ}\right.$ rotation) rather than up $\left(0^{\circ}\right.$ presentation $)$, the authors concluded that their subject had some cortical representations of hands and feet. The ways in which these representations had been built up in the first line remain obscure. Yet, on the phenomenal level, they would manifest themselves as phantom limbs. This is in fact what makes the issue of presence/ absence of phantom limbs in bilateral amelia so crucial for investigations of motor imagery (Price, 2006, for overview): decades of a vivid presence of a pair of hands are unlikely to remain without effect on hand motor imagery. A second study investigated hand laterality decisions in three individuals with unilateral hand amelia (Nico et al., 2004). A MOLA was found for the existing, but not for the missing limb. Because of the evidence suggesting that capacity for hand movement is important to the formation of cortical representations of hand position, the nature of such representations in subjects born without hands is of considerable interest. Data from a total of three reported amelic individuals do not enable firm conclusions on this matter, especially not in the absence of any information about phantom limb status. Therefore, we undertook the present study of mental hand rotation in 14 subjects with unilateral amelia and 2 subjects with bilateral amelia. Three main predictions were made: (1) Based on the preliminary data by Nico et al. (2004; three individuals with left-hand amelia), we hypothesized faster RTs for the preserved compared with the absent hand; (2) Because of previous reports of a strong bimanual coupling in unilateral hand amputees (Franz \& Ramachandran, 1998), we expected a MOLA for both the preserved and the absent hand. In addition to the individuals with unilateral hand amelia, we also examined two individuals born with bilaterally missing limbs. One of these individuals (A.Z.) reported vivid phantom sensations while the other (C.L.) had never experienced any. Previous work with these two individuals described that the one with phantoms, but not the one without, showed intact visual-handmotor interactions (Funk et al., 2005a). Therefore, we expected, (3) A.Z., but not C.L., to evidence a pattern of hand laterality decisions similar to the one displayed by normally limbed participants.

\section{METHODS}

\section{Research Participants}

Fourteen subjects with one hand missing since birth (hereafter referred to a: "unilaterally amelic participants"), 2 subjects with congenital absence of both hands (hereafter "bilaterally amelic participants"), and 24 normally limbed control subjects participated in the current study that had been approved by the local Ethics Committee. Written informed consent was obtained from every participant. No participant had ever suffered from serious health problems or developmental disorders as assessed by a standardized inventory (Campbell, 2000). All participants scored in the normal range in a paper-pencil test of mental object rotation (Thurstone \& Thurstone, 1941/1962). This finding means that any performance differences in the hand laterality task could be interpreted as due to differences in presence/absence of hands, rather than some generally impaired ability in mental rotation. Moreover, a difference in the hand laterality task could be interpreted as a deficit in motor imagery. Participants were recruited by flyers, personal contact, or by a self-help group for amelic individuals.

Preliminary to this study, the amelic participants were administered a mental rotation task with nonbody related objects, that is, cars, as stimuli (Funk et al., 2005b). Hoods pointed left, right, up, or down, and the task was to indicate, whether the presented car would drive to the left or to the right side if mentally rotated into a horizontal position (i.e., wheels down). A right-sided motor response (by a hand, stump, or prosthesis) was required for right-driving cars and a left-sided response for left-driving cars. We administered this task to ascertain that the physical attributes of their upper limbs would not systematically influence a motor response.

In the unilaterally amelic participants, responding with a stump or a prosthesis was statistically comparable to responding with the normally developed hand, both with respect to accuracy and speed. In the bilaterally amelic participants, responding with upper arm stumps or with the right foot did not introduce any motor bias for one or the other response key.

\section{Unilaterally amelic participants}

Nine unilaterally amelic participants (five women) with an absent left hand (hereafter "absL"; age range 8 to 33 years; mean $=17.4$ years, $S D=8.7$ years) and five unilaterally amelic participants ( 2 women) with an absent right hand (hereafter "absR"; age range 12 to 27 years, mean $=20.6$ years, $S D=5.7$ years) were examined. A hand was regarded as missing if all fingers were absent. No member of the unilaterally amelic group had ever experienced any phantom sensations of the missing limb during waking life (Brugger \& Regard, 1998). However, some had experienced themselves performing bimanual activities during sleep dreams. Table 1 gives the age and gender of the amelic participants, side and extent of amelia, as well as a summary of the subject's use (if any) of a prosthesis.

The medical cause of the amelia was unknown in every instance. Figure 1A displays a participant with a missing left hand performing the hand laterality task.

\section{Bilaterally amelic participants}

Two subjects with bilaterally missing hands participated in the present study. A.Z. is a university-educated woman, born in 1953 without lower arms and lower legs. A.Z. experienced phantom sensations of her missing body parts as long as she can remember. C.L. is a journalist born in 1962 with- 
Table 1. List of amelic participants with their individual characteristics

\begin{tabular}{lclccc}
\hline \hline Side of hand amelia & Extent of amelia & Prosthesis & Phantoms & $\begin{array}{c}\text { Age } \\
(\text { yr })\end{array}$ & Gender \\
\hline Right & Below elbow & Cosmetic & No & 27 & $\mathrm{M}$ \\
Right & Above elbow & None & No & 12 & $\mathrm{~F}$ \\
Right & Above elbow & None & No & 23 & $\mathrm{~F}$ \\
Right & Below elbow & None & No & 18 & $\mathrm{M}$ \\
Right & Below elbow & Cosmetic & No & 23 & $\mathrm{M}$ \\
Left & Below elbow & Myoelectric & No & 11 & $\mathrm{~F}$ \\
Left & Above elbow & None & No & 8 & $\mathrm{M}$ \\
Left & Below elbow & None & No & 10 & $\mathrm{~F}$ \\
Left & Below elbow & None & No & 12 & $\mathrm{~F}$ \\
Left & Below elbow & Cosmetic & No & 12 & $\mathrm{~F}$ \\
Left & Below elbow & Cosmetic & No & 22 & $\mathrm{~F}$ \\
Left & Below elbow & Myoelectric & No & 26 & $\mathrm{M}$ \\
Left & Below elbow & Myoelectric & No & 33 & $\mathrm{M}$ \\
Left & Below elbow & Myoelectric & No & 23 & $\mathrm{M}$ \\
Right and left (A.Z.) & Above elbow & None & Yes & 50 & $\mathrm{~F}$ \\
Right and left (C.L.) & Above elbow & None & No & 41 & $\mathrm{M}$ \\
\hline \hline
\end{tabular}

out arms and shortened legs due to thalidomide-related embryopathy. In daily life, he uses his right foot for writing, eating, pointing, and gesturing. He has never experienced any phantom sensations of his missing limbs. More detail to these two participants' physical appearance can be found in Brugger and Funk (2007), Brugger et al. (2000), and Funk et al. (2005a). Figures 1B and 1C show them performing the hand laterality task.

\section{Normally limbed participants}

A total of 24 normally limbed adults, 12 men and 12 women, formed the control group (mean $=31.7$ years, $S D=8.32$ years). Most control participants were students or had already received an academic degree. All 24 members of the control group were right-handed according to the 13-item inventory of Chapman and Chapman (1987), and none had ever had a psychiatric or neurological illness nor suffered from developmental disorders (Campbell, 2000).

\section{Measures}

\section{Hand laterality task}

Photographs of human hands (50\% left and 50\% right hands; $50 \%$ back views and $50 \%$ palm views; 4 angles of rotation: $0^{\circ}, 90^{\circ}, 180^{\circ}$, and $270^{\circ}$, clockwise along the vertical axis) were presented on a portable computer with an LCD monitor in the center of the screen. $0^{\circ}$ corresponds to a hand with fingers pointing upward, $180^{\circ}$ corresponds to a hand with fingers pointing downward. See Figure 2 for four sample hand stimuli. Maximal horizontal and vertical extension of a stimulus was $11.3^{\circ}$ of visual angle. We decided to use only four stimulus orientations because of concerns about too lengthy testing (especially for the children, whose inclusion enabled testing of the sizable sample of unilaterally amelic participants introduced here). Moreover, inclusion of more angular rotations in hand laterality tasks would have been unlikely to produce a fundamentally different pattern of results.

Participants were required to decide as fast and correctly as possible whether a presented hand stimulus depicted a right or a left hand. "RIGHT" ("LEFT") decisions had to be provided by pressing a right-sided (left-sided) key on a two-key special purpose keyboard with the right (left) hand, stump or prosthesis. The exact distance between body and keyboard was adjusted in a way that allowed all participants to reach the two keys comfortably and with a comparable angle between upper and lower arm. CL used the same keyboard, but pressed the response keys with his right foot
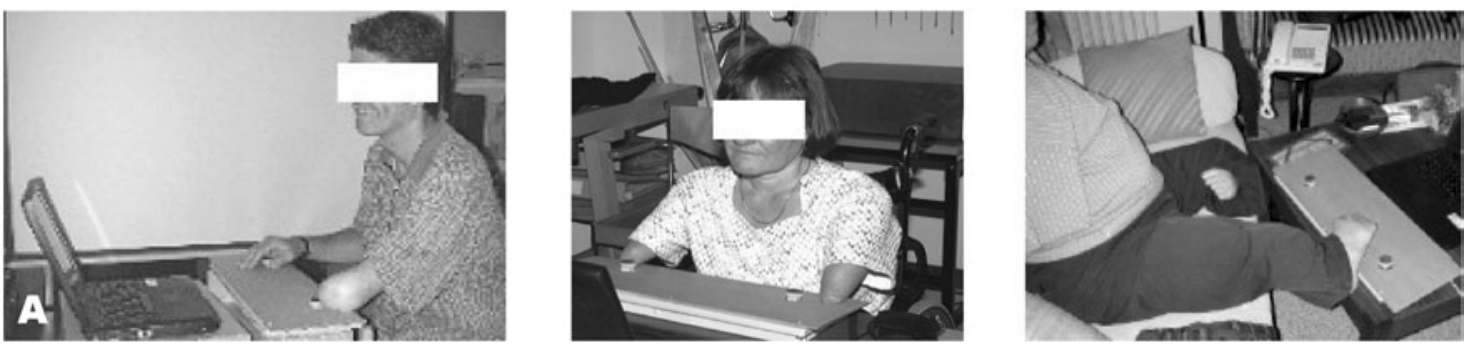

Fig. 1. A participant with a unilaterally missing left hand (A), participant A.Z. with a tetra-amelia (B), and C.L. with a bilateral hand amelia (C). 


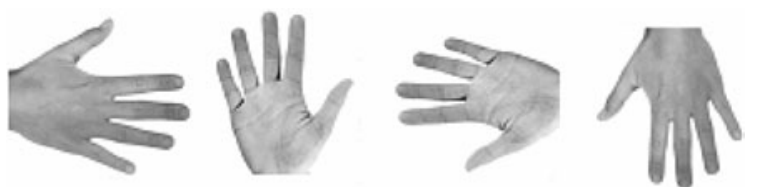

Fig. 2. Shown are 4 of 16 stimulus samples used. Displayed are, from left to right, angles of $90^{\circ}, 0^{\circ}, 270^{\circ}$, and $180^{\circ}$. A total of $50 \%$ percent of the stimuli represented left hands, $50 \%$ right hands. Orthogonally, $50 \%$ of the hands were presented in palm view, $50 \%$ in back view.

(initiating both left-sided and right-sided responses from a location in between the two keys; Fig. 1C). The two response keys could be adjusted in height to be optimally comfortable for each individual subject. Stimulus presentation and response collection were controlled by SuperLab software Pro Version 2.0 (Cedrus Corporation, 2002).

\section{PROCEDURE}

Normally limbed and unilaterally amelic participants responded to 64 randomized stimuli (4 repetitions of the 16 different stimuli). Bilaterally amelic participants responded to 160 randomized stimuli (10 repetitions of the 16 different stimuli). The higher number of repetitions in the bilaterally amelic subjects was to enhance the statistical power of the single case analyses. We knew that these subjects would tolerate the corresponding number of stimulations. We administered fewer stimuli to unilaterally amelic subjects, six of whom were children, so as not to risk losing subjects because of excessive study duration. There are no reasons to assume that fewer trials would lead to principally different pattern of results. As the unilaterally amelic subjects' results were analyzed on a group level, no more repetitions were required. There were 16 practice trials (discarded from the analysis).

Stimulus exposure was only terminated by the participants' response. During the task a dark cloth was covering hands and underarms (or prostheses, respectively) to prevent a visual image of the own limbs or limb-substitutes.

\section{RESULTS}

Data were transformed logarithmically due to their skewed distribution. As assessed by Kolmogorov-Smirnov statistics, the thus transformed data were normally distributed. All participants performed significantly above chance (i.e., $>90$ correct responses for participants receiving 160 trials and $>40$ correct responses for the participants receiving 64 trials, 5\% $\alpha$ level in each case). There was no speedaccuracy trade off (correlation $Z$ test, $r=-.22 ; p=.29$ ), and we report analyses of RTs of correct responses.

\section{Normally Limbed Participants}

We calculated a four-way repeated measures analysis of variance (ANOVA) of the RTs of correct responses, with gender as a between-subjects factor and stimulus laterality (i.e., side of the presented hand as variate, right, or left), view (palm or back of hand), and angle of rotation $\left(0^{\circ}, 90^{\circ}\right.$, $180^{\circ}, 270^{\circ}$; clockwise) as within-subject factors. Significant factors and interactions are listed in Table 2, along with the nature of the differences.

Focusing on the MOLA (see Figure 3), we note that both right and left hand stimuli were significantly faster responded to when presented in a medial (i.e., $270^{\circ}$ for right hands and $90^{\circ}$ for left hands) as compared to a lateral orientation (i.e., $90^{\circ}$ for right and $270^{\circ}$ for left hands): right hands: $t(23)=$ $3.84, p<.001$; left hands: $t(23)=3.69, p<.001$.

\section{Unilaterally Amelic Participants}

A five-way repeated measures ANOVA of the RTs of correct responses (between-subjects factors gender and side of missing limb, within-subject factors stimulus laterality (side of visually presented hand), view and angle of rotation) was conducted. For significant factors and interactions see Table 2 .

absR showed a significant MOLA for the right hand [90 vs. $270^{\circ}: t(4)=2.35, p<.05$ ], but not for the preserved left hand [ $90^{\circ}$ vs. $\left.270^{\circ}: t(4)=1.15, p=.15\right]$. absL showed a significant MOLA for the preserved, but also for the missing hand [right hands $90^{\circ}$ vs. $270^{\circ}: t(8)=2.7, p<.05$, left hands $90^{\circ}$ vs. $270^{\circ}: t(8)=2.3 ; p<.05$ ]; see Figure 4 .

Checking for a potential influence of absence/presence of prosthesis on RTs, we calculated a four-way repeated measure ANOVA of the RTs of correct responses with the between-subjects factor prosthesis (myoelectric, cosmetic, no) and within-subject factors stimulus laterality, view, and angle of rotation. The factor prosthesis $[F(2,11)=1.78$; $p=.21]$ and all interactions with the other factors were not significant.

\section{Bilaterally Amelic Participants}

A.Z.'s and C.L.'s RTs of correct decisions were subject to an ANOVA with the factors stimulus laterality, view, and angle of rotation. Significant main effects and interactions are presented in Table 2.

Evidently, A.Z. showed a MOLA for right [t(37) $=2.63$, $p<.01]$ and left $[t(38)=2.01, p<.05]$ hand stimuli (see Figure 5, left panel). C.L.'s RTs to right and to left hands did not differ between the four angles of rotation. Consequently, there was no significant MOLA, neither for left nor for right hands (Figure 5, right panel).

\section{DISCUSSION}

We administered a hand laterality task to 14 subjects with unilateral hand amelia, to two subjects with bilateral hand amelia, and to 24 normally limbed control subjects. We investigated whether the physical presence of a hand is necessary for the visual-somesthetic interactions typically demonstrated by normally limbed subjects in this type of task. 
Table 2. List of the significant main effects and interactions as well as the nature of the difference in normally limbed participants (NLP), unilaterally amelic participants (UAP), and the bilaterally amelic participants A.Z. and C.L.

\begin{tabular}{|c|c|c|c|}
\hline Group & $\begin{array}{l}\text { Significant main effects } \\
\text { and interactions }\end{array}$ & $F$ value $/ p$ value & Nature \\
\hline NLP & Angle of rotation & $F(3,60)=62.55, p<.001$ & $\begin{array}{l}\text { RTs increased with hand rotation away from } 0^{\circ} \\
(p<.001)\end{array}$ \\
\hline NLP & View & $F(1,20)=30.53, p<.001$ & Backs of hands faster than palms $(p<.001)$ \\
\hline NLP & Stimulus laterality & $F(1,20)=20.62, p<.001$ & Right hands faster than left hands $(p<.001)$ \\
\hline NLP & View $\times$ angle of rotation & $F(3,60)=13.67, p<.001$ & $\begin{array}{l}\text { Backs of hands faster than palms in } 0^{\circ}, 90^{\circ} \text { and } 270^{\circ} \\
(p<.001)\end{array}$ \\
\hline NLP & Stimulus laterality $\times$ angle of rotation & $F(3,60)=9.80, p<.001$ & $\begin{array}{l}\text { Right hands faster than left hands in } 0^{\circ}, 180^{\circ} \text { and } \\
270^{\circ}(\mathrm{p}<.01) \text { left hands faster in } 90^{\circ}(p<.05) \text {; } \\
\text { see Figure } 3\end{array}$ \\
\hline NLP & \multicolumn{3}{|c|}{ Three-way interaction between view $\times$ stimulus laterality $\times$ angle of rotation; not further discussed } \\
\hline UAP & Angle of rotation & $F(3,30)=40.98, p<.001$ & $\begin{array}{l}\text { RTs increased with hand rotation away from } 0^{\circ} \\
(p<.05)\end{array}$ \\
\hline UAP & View & $F(1,10)=32.81, p<.001$ & Backs of hands faster than palms $(p<.001)$ \\
\hline UAP & Stimulus laterality $\times$ missing limb & $F(1,10)=31.22, p<.001$ & $\begin{array}{l}\text { absL }(p<.01) \text { and absR }(p=.01) \text { faster } \\
\text { recognized hand stimuli corresponding to their } \\
\text { normally developed limb than to those corresponding } \\
\text { to their missing limb }\end{array}$ \\
\hline UAP & View $\times$ angle of rotation & $F(3,30)=6.52, p<.01$ & $\begin{array}{l}\text { RTs of palms and backs of hands increased with angular } \\
\text { rotation of the hand stimuli away from } 0^{\circ} / 360^{\circ} \text {. }\end{array}$ \\
\hline UAP & Stimulus laterality $\times$ angle of rotation & $F(3,30)=6.95, p=.001$ & $\begin{array}{l}\text { Right hands }=\text { left hands in } 90^{\circ}(p=.07) \\
\text { Right hands faster than left hands in } 270^{\circ}(p<.01) \\
\text { RTs to right and left hands increased with angular } \\
\text { rotation away from } 0^{\circ} / 360^{\circ}(p<.001) \text {; see Figure } 4\end{array}$ \\
\hline UAP & \multicolumn{3}{|c|}{ Interaction between view, stimulus laterality and side of missing limb; not further discussed } \\
\hline A.Z & Angle of rotation & $F(3,141)=7.33, p<.01$ & $\begin{array}{l}\text { RTs increased with hand rotation away from } 0^{\circ} \\
(p<.01)\end{array}$ \\
\hline A.Z. & View $\times$ angle of rotation & $F(3,141)=4.31, p<.01$ & Palms faster than backs of hands in $180^{\circ}(p=.001)$ \\
\hline A.Z. & Stimulus laterality $\times$ angle of rotation & $F(3,141)=3.90, p=.01$ & $\begin{array}{l}\text { Right faster than left hands in } 270^{\circ}(p<.01) \\
\text { RTs to right and left hands increased with angular } \\
\text { rotation away from } 0^{\circ} \text {; see Fig. } 5 \text {, left panel }\end{array}$ \\
\hline C.L. & Angle of rotation & $F(3,129)=3.83, p=.01$ & RTs to hands under $180^{\circ}$ longer than $270^{\circ}(p<.05)$ \\
\hline C.L. & View & $F(1,129)=41.2, p<.001$ & Backs of hands faster than palms $(p<.001)$ \\
\hline
\end{tabular}

Note. RTs, reaction times; absL, absent left hand; absR, absent right hand.

These interactions comprise, first, a superior performance for pictures of hands corresponding to the subject's dominant hand, at least for right-handed populations; second, a back-over-palm view advantage for hands presented with fingers pointing up and to either side, but not for those with fingers pointing down; third, a MOLA of the RTs of correct decisions, that is, faster responses to hands with fingers pointing toward as compared to away from the body's midsagittal plane. All these previously reported effects could be replicated in the present population of normally limbed control subjects (see Table 3), and once again support the view that (right) handedness and, more generally, a lifelong use of hands are important determinants of left-right decisions regarding visually presented hands.

The 14 participants with only one hand missing showed as clear a back-over-palm recognition advantage as did normally limbed subjects. Also, and again in correspondence with the data from the control group, RTs of correct decisions were longer for $90^{\circ} / 270^{\circ}$ and still longer for $180^{\circ}$ rotations of a stimulus hand from a canonical, $0^{\circ}$ fingers-up orientation. According to our original prediction (1), the life-long physical absence of one hand manifested itself in the fact that hand stimuli depicting the preserved hand (a left hand for subjects of the absR group and a right hand for 


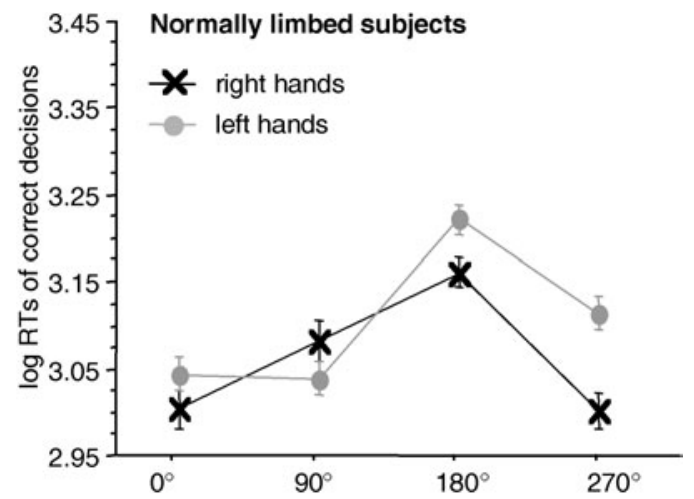

Fig. 3. Significant interaction between stimulus laterality and angle of rotation in the participants of the normally limbed control group. Displayed are means \pm standard errors. There is a significant "medial-over-lateral advantage" (MOLA), that is, faster decisions to medially as compared to laterally displayed hands. RTs, reaction times.

the absL group) were significantly faster recognized than pictures of a hand corresponding to the missing one. This effect cannot be an artifact of peripheral factors (response initiated by stump or prosthesis versus response initiated by preserved hand), because manual responses were comparable to stump/prosthesis responses as long as the stimuli were left or right driving cars (our control experiment).

In accordance with our prediction (2), the participants with only a right hand showed a MOLA not only for hand pictures representing their normally developed limb, but also for those depicting the hand they had never felt, seen, nor used for any action. This finding is compatible with evidence for strong bimanual coupling in (traumatic) arm amputees (Franz \& Ramachandran, 1998), although those subjects had experienced phantom sensations of their absent hand. It contrasts, however, with data reported by Nico et al.
(2004), whose three amelic individuals showed a MOLA for the right (present), but not the left (congenitally absent) hand. These authors' conclusion that congenital limb absence "precludes the ability to produce joint-constrained mental simulations for the deleted hand" (p. 128) may have been unwarranted as it was based on the data from not enough participants. Unexpectedly, in our five individuals born with only a left hand, a significant MOLA emerged exclusively for pictures of the absent right hand. However, the trend favors also a MOLA for left hands even though the difference did not reach statistical significance (possibly due to the small number of subjects). As these individuals thus showed a pattern of MOLAs similar to the one observed in healthy controls (significant for right hands, only tendentially present for left hands; see Figure 3), we speculate that most of these individuals would have turned out to be righthanded, and that this latent, "presumptive right-handedness" was more decisive than the longstanding use of the left hand.

Wearing a prosthesis, independent of its type (cosmetic or myoelectric), did not influence the unilaterally amelic individuals' performance. In the investigation by Nico et al. (2004), traumatic amputees' motor imagery was impaired by prosthesis use. The impairment reported by these authors was less pronounced if the prosthesis was of a myoelectric type and could arguably be used as a tool and thus incorporated in the body schema. Evidently, tool-use in cases of amputation bears more capacity for functional reorganization than in cases of congenital absence of a limb.

We had originally planned to study unilaterally amelic participants' performance in the hand laterality task as a function of phantom status, i.e., whether or not they had experienced phantom sensations of their absent limb (we had predicted a stronger MOLA for an absent hand with compared to without phantom sensations). However, despite careful, nonsuggestive questioning (Brugger \& Funk, 2007;
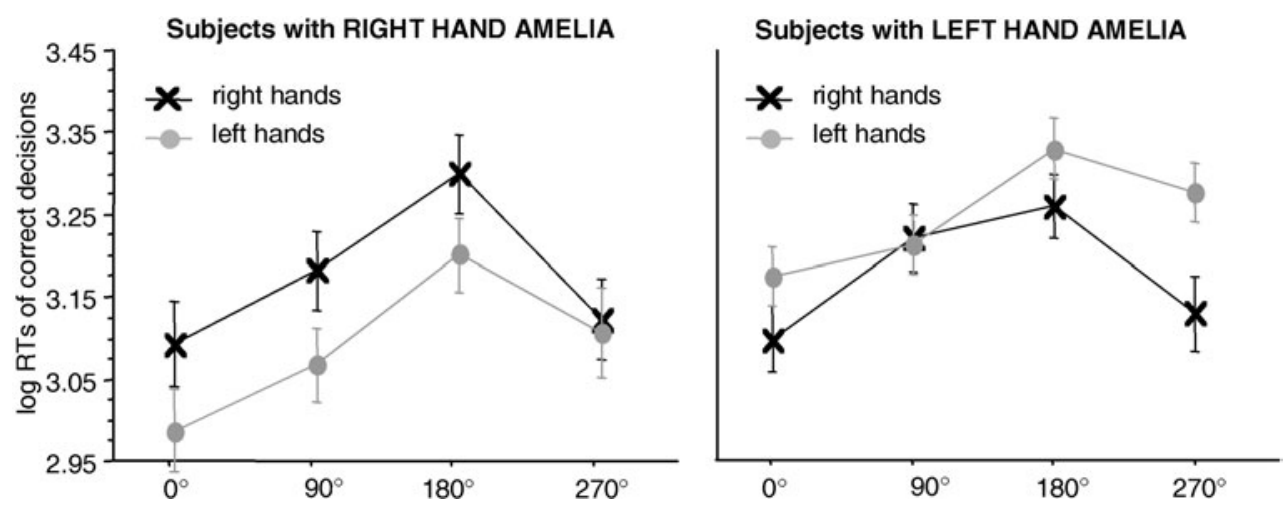

Fig. 4. Significant interaction between stimulus laterality and angle of rotation in the participants with a unilateral hand amelia. The left panel displays the results of the participants with a missing right hand, the right panel the results of the participants with a missing left hand. Displayed are means \pm standard errors. Participants with a congenitally missing right hand show a significant "medial-over-lateral advantage" (MOLA), that is, faster decisions to medially as compared to laterally displayed hands for right hand stimuli. Participants with a congenitally missing left hand show a significant MOLA for right and left hand stimuli. RTs, reaction times. 

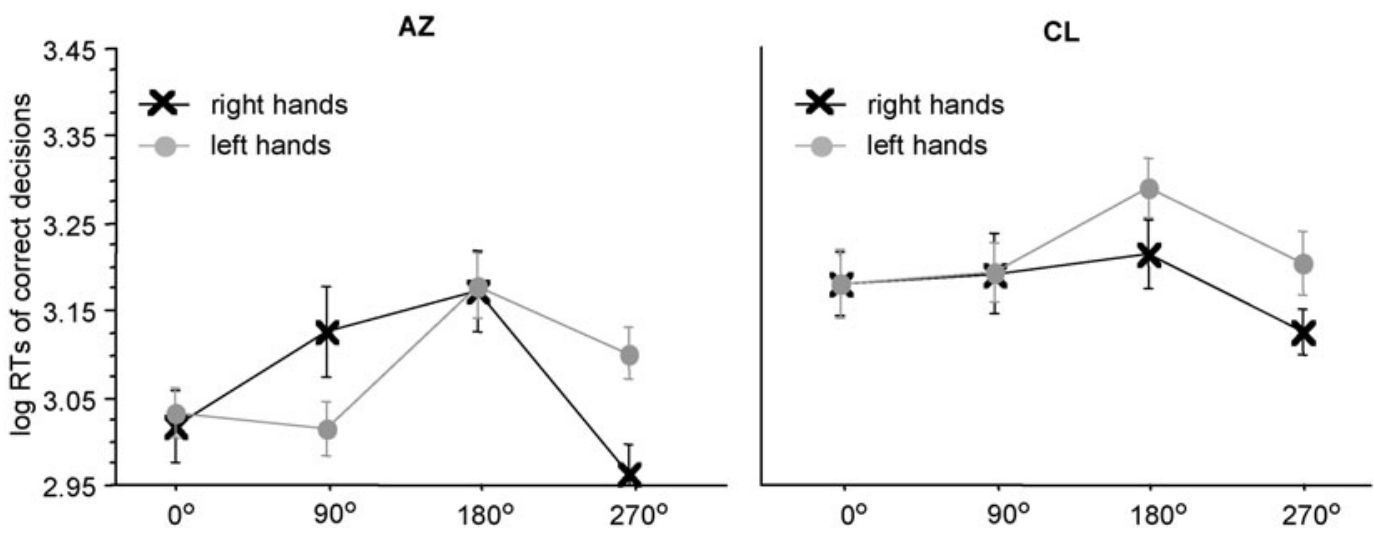

Fig. 5. Significant interaction between stimulus laterality and angle of rotation in the participants with a bilateral hand amelia, the left panel displays the results of participant A.Z. with phantom sensations, the right panel the results of participant C.L. without phantom sensations. Displayed are means \pm standard errors. The "medial-over-lateral advantage" (MOLA), that is, faster decisions to medially compared to laterally displayed hands, is significant for A.Z., but not for C.L.

Valentin, 1836), we could not find one single unilaterally amelic individual with phantoms in our study population.

For these reasons, the data provided by the participants A.Z. and C.L. deserve special attention. As these two subjects have never owned even one single hand, any effects of implicit motor imagery as reflected in the performance in hand laterality tasks might tell us about the components of body schema that were not acquired by habitual physical execution of hand movements. To facilitate our discussion of these subjects' performance, Table 3 summarizes the main effects and interactions found in the individual analyses.

As is evident from Table 3 and Figure 5, both participants with congenital absence of both hands showed a main effect for angle of rotation: responses to hands with fingers pointed downward $\left(180^{\circ}\right)$ were slower. That only one of these subjects had phantom sensations (A.Z.) suggests that phantom sensations do not underlie this angle of rotation effect.
Neither A.Z. nor C.L. showed a preference in recognizing right or left hands. One could have expected an advantage in recognizing right over left hands as reported from right-handed, normally limbed subjects, for A.Z. as she previously provided evidence reflecting "right-handedness" (Brugger \& Funk, 2007). As C.L. uses his right foot for all daily activities, we could have expected a right hand advantage in his data as well. C.L., however, responded equally fast to right and left hands. We speculate that a recognition advantage for right hands may thus be dependent on the use of a real hand.

Most important in the context of this study was the evaluation of the interaction between stimulus laterality and angle of rotation of which the MOLA is the crucial aspect. According to our third prediction, we found significant MOLAs for both hands for A.Z., but not for C.L. While the absence of any MOLA in the case of C.L. speaks for the necessity of sensorimotor experiences with reference to

Table 3. Summary of significant main effects and interactions (+: present; -: absent) found in the three-way analysis of variance of the reaction times of correct decisions in the hand laterality task with the factors angle of rotation $\left(0^{\circ}, 90^{\circ}, 180^{\circ}, 270^{\circ}\right)$, stimulus laterality (left or right hand), and view (back of hands or palm)

\begin{tabular}{|c|c|c|c|c|c|c|c|}
\hline \multirow[b]{2}{*}{ Subjects } & \multicolumn{3}{|c|}{ Main effects: } & \multicolumn{4}{|c|}{ Interactions: } \\
\hline & $\begin{array}{c}\mathrm{I}: \\
\text { angle of } \\
\text { rotation }\end{array}$ & $\begin{array}{c}\text { II: } \\
\text { stimulus } \\
\text { laterality }\end{array}$ & $\begin{array}{l}\text { III: } \\
\text { view }\end{array}$ & $\mathrm{I} \times \mathrm{II}$ & II $\times$ III & $\mathrm{I} \times \mathrm{III}$ & $\mathrm{I} \times \mathrm{II} \times \mathrm{III}$ \\
\hline A.Z.; with phantoms & + & - & - & + & - & + & - \\
\hline C.L.; without phantoms & + & - & + & - & - & - & - \\
\hline $\begin{array}{l}\text { Normally limbed subjects (in accordance with } \\
\text { published findings, e.g., Parsons, 1987) }\end{array}$ & + & + & + & + & - & + & + \\
\hline
\end{tabular}

Note. Data from A.Z., C.L., and normally limbed subjects. 
hands, its presence in A.Z. refutes this contention. We speculate that A.Z.'s vivid phantoms of fingers, hands, and arms, reportedly characterized by a movement repertoire corresponding to that of normal upper limbs (Funk et al., 2005a), enabled her to boost her mental imagery. ${ }^{1}$

In summary, our experiment revealed that subjects' performance on a hand laterality task is clearly influenced by the congenital absence of a hand. A handedness effect was found in unilaterally amelic subjects, indicating that, compared to the developed hand, the absent hand was more difficult to classify as a left or a right limb. In addition, however, also decisions regarding the absent hand were dependent on the display angle of a stimulus, suggesting some spared motor imagery capacity. Finally, in the two participants born with neither a left nor a right hand, no handedness effects were evident. In the one person with a history of phantom sensations, but not in the one without, RTs to stimulus hands depicted in, for normally limbed subjects, "awkward" rotation angles were longer that those to "comfortable" hand postures, indicating a motor system participation. We speculate that the presence of phantom sensations may be an important factor in the motor imagery regarding congenitally absent limbs. We readily admit that the present findings cannot directly elucidate the mechanisms that lead to the emergence of phantoms of congenitally absent limbs. As long as not more persons born with hand amelia and phantom sensations will be available, the field will probably remain plagued with speculations (Price, 2006; see also footnote 1).

\section{ACKNOWLEDGMENTS}

We thank the people from the organization Pinocchio (www.pinocchio.ch) for their help in subject recruitment and the participants in the present experiments for their invaluable cooperation. This work was supported by the Swiss National Science Foundation (grant no. 3100-67168.01 to P.B.).

\section{REFERENCES}

Bonda, E., Petrides, M., Frey, S., \& Evans, A. (1995). Neural correlates of mental transformations of the body-in-space. Proceedings of the National Academy of Sciences of the United States of America, 92, 11180-11184.

Brugger, P. \& Funk, M. (2007). Out on a limb: Neglect and confabulation in the study of aplasic phantoms. In S. della Sala

${ }^{1}$ An anonymous referee of this paper argued differently. The absence of a MOLA in C.L. (Fig. 5, right panel) was interpreted as a type II error, an interpretation that diminishes the importance of the factor presence/ absence of aplasic phantoms. In view of the small number of subjects tested, we admit that our speculations about a causal role of phantom limb experience for motor imagery bears the potential of a type I error. The history of clinical-scientific research on phantoms of congenitally absent limbs is a key lecture to illustrate the interplays between type I and type II errors in a field that offers relatively scarce data sources (see Brugger and Funk, 2007, for extensive discussion).
(Ed.), Tall Tales about the Mind and Brain. Separating fact from fiction (pp. 348-368). Oxford: Oxford University Press.

Brugger, P., Kollias, S.S., Müri, R.M., Crelier, G., Hepp-Reymond, M.-C., \& Regard, M. (2000). Beyond re-membering: Phantom sensations of congenitally absent limbs. Proceedings of the National Academy of Sciences of the United States of America, 97, 6167-6172.

Brugger, P. \& Regard, M. (1998). Phantom limbs in congenital tetramelia: A case report. Journal of the International Neuropsychological Society, 4, 206.

Campbell, J.J. (2000). Neuropsychiatric assessment. In C.E. Coffey \& J.L. Cummings (Eds.), Textbook of Geriatric Neuropsychiatry (2nd ed., pp. 109-124). Washington, DC: American Psychiatric Press.

Cedrus Corporation. (2002). SuperLab Pro (Version 2.0) [Computer software]. San Pedro, CA: Cedrus Corporation.

Chapman, L.J. \& Chapman, J.P. (1987). The measurement of handedness. Brain and Cognition, 6, 175-183.

Decety, J., Perani, M., Jeannerod, V., Bettinardi, B., Tadary, R., Woods, J., Mazziotta, C., \& Fazio, F. (1994). Mapping motor representations with positron emission tomography. Nature (London), 371, 600-602.

Fiorio, M., Tinazzi, M., \& Aglioti, S.M. (2006). Selective impairment of hand mental rotation in patients with focal hand dystonia. Brain, 129, 47-54.

Franz, E.A. \& Ramachandran, V.S. (1998). Bimanual coupling in amputees with phantom limbs. Nature Neuroscience, 1 , 443-444.

Funk, M., Shiffrar, M., \& Brugger, P. (2005a). Hand movement observation by individuals born without hands: Phantom limb experience constrains visual limb perception. Experimental Brain Research, 164, 341-346.

Funk, M., Wilkening, F., \& Brugger, P. (2005b). Motor processes in children's imagery: The case of mental rotation of hands. Developmental Science, 8, 402-408.

Gentilucci, M., Daprati, E., \& Gangitano, M. (1998). Righthanders and left-handers have different representations of their own hand. Cognitive Brain Research, 6, 185-192.

Johnson, S.H., Sprehn, G., \& Saykin, A.J. (2002). Intact motor imagery in chronic upper limb hemiplegics: Evidence for activity-independent action representations. Journal of Cognitive Neuroscience, 14, 841-852.

Kosslyn, S.M., Digirolamo, G.J., Thompson, W.L., \& Alpert, N.M. (1998). Mental rotation of objects versus hands: Neural mechanisms revealed by positron emission tomography. Psychophysiology, 35, 151-161.

Nico, D., Daprati, E., Rigal, F., Parsons, L., \& Sirigu, A. (2004). Left and right hand recognition in upper limb amputees. Brain, 127, 120-132.

Parsons, L.M. (1987). Imagined spatial transformations of one's hands and feet. Cognitive Psychology, 19, 178-241.

Parsons, L.M. (1994). Temporal and kinematic properties of motor behavior reflected in mentally simulated action. Journal of Experimental Psychology: Human Perception and Performance, 20, 709-730.

Parsons, L.M., Fox, P.T., Downs, J.H., Glass, T., Hirsch, T.B., Martin, C.C., Jerabek, P.A., \& Lancaster, J.L. (1995). Use of implicit motor imagery for visual shape discrimination as revealed by PET. Nature (London), 375, 54-58.

Price, E.H. (2006). A critical review of congenital phantom limb cases and a developmental theory for the basis of body image. Consciousness and Cognition, 15, 310-322. 
Sekiyama, K. (1982). Kinesthetic aspects of mental representations in the identification of left and right hands. Perception and Psychophysics, 32, 89-95.

Shenton, J.T., Schwoebel, J., \& Coslett, H.B. (2004). Mental motor imagery and the body schema: Evidence for proprioceptive dominance. Neuroscience Letters, 370, 19-24.
Thurstone, L.L. \& Thurstone, T.G. (1941/1962). Primary Mental Abilities. Chicago: Science Research Associates.

Valentin, G. (1836). Über die subjektiven Gefühle von Personen, welche mit mangelhaften Extremitäten geboren sind. Repertorium für Anatomie und Physiologie, 1, 328-337. 